\title{
Cotargeting MNK and MEK kinases induces the regression of NF1-mutant cancers
}

\author{
Rebecca Lock,, ${ }^{1,2}$ Rachel Ingraham, ${ }^{1,2}$ Ophélia Maertens, ${ }^{1,2}$ Abigail L. Miller, ${ }^{1,2}$ Nelly Weledji, ${ }^{1,2}$ Eric Legius, ${ }^{3,4}$ Bruce M. Konicek, ${ }^{5}$ \\ Sau-Chi B. Yan, ${ }^{5}$ Jeremy R. Graff, ${ }^{6}$ and Karen Cichowski ${ }^{1,2,7}$ \\ 'Cenetics Division, Department of Medicine, Brigham and Women's Hospital, Boston, Massachusetts, USA. ${ }^{2 H a r v a r d ~ M e d i c a l ~ S c h o o l, ~ B o s t o n, ~ M a s s a c h u s e t t s, ~ U S A . ~}{ }^{3}$ Center for Human Genetics, University \\ Hospital Leuven, Leuven, Belgium. ${ }^{4}$ Department of Human Genetics, Catholic University Leuven, Leuven, Belgium. ${ }^{5}$ Eli Lilly and Co., Lilly Corporate Center, Indianapolis, Indiana, USA. \\ ${ }^{6}$ Biothera Pharmaceuticals Inc., Eagan, Minnesota, USA. 'Ludwig Center at Dana-Farber/Harvard Cancer Center, Boston, Massachusetts, USA.
}

\begin{abstract}
Neurofibromin 1-mutant (NF1-mutant) cancers are driven by excessive Ras signaling; however, there are currently no effective therapies for these or other Ras-dependent tumors. While combined MEK and mTORC1 suppression causes regression of NF1-deficient malignancies in animal models, the potential toxicity of cotargeting these 2 major signaling pathways in humans may necessitate the identification of more refined, cancer-specific signaling nodes. Here, we have provided evidence that MAPK-interacting kinases (MNKs), which converge on the mTORC1 effector elF4E, are therapeutic targets in NF1-deficient malignancies. Specifically, we evaluated primary human NF1-deficient peripheral nervous system tumors and found that MNKs are activated in the majority of tumors tested. Cenetic and chemical suppression of MNKs in NF1-deficient murine tumor models and human cell lines potently cooperated with MEK inhibitors to kill these cancers through effects on elF4E. We also demonstrated that MNK kinases are important and direct targets of cabozantinib. Accordingly, coadministration of cabozantinib and MEK inhibitors triggered dramatic regression in an aggressive genetically engineered tumor model. The cytotoxicity of this combination required the suppression of MNK-induced elF4E phosphorylation and was not recapitulated by suppressing other cabozantinib targets. Collectively, these studies demonstrate that combined MNK and MEK suppression represents a promising therapeutic strategy for these incurable Rasdriven tumors and highlight the utility of developing selective MNK inhibitors for these and possibly other malignancies.
\end{abstract}

\section{Introduction}

Loss-of-function mutations in the tumor suppressor neurofibromin 1 (NF1) underlie the common familial cancer syndrome neurofibromatosis type 1 and promote the development of a variety of sporadic cancers including glioblastoma, melanoma, and non-small cell lung cancer (NSCLC) (1). The NF1 gene encodes a Ras GTPase-activating protein (RasGAP) (2, 3). Accordingly, excessive Ras signaling underlies the pathogenesis of NF1-mutant tumors (4). However, despite this insight, there are currently no effective treatments for any NF1-deficient or Ras-driven cancers.

Because NF1-mutant tumors are driven by excessive Ras signaling, we and others have been evaluating Ras pathway inhibitors in a variety of tumor models (5-7). Fortunately, MEK inhibitors exhibit efficacy in mouse models of clinically important benign tumors associated with neurofibromatosis type 1 , including nervous system lesions known as neurofibromas as well as juvenile myelomonocytic leukemia (JMML) $(6,7)$, which has inspired the development of several clinical trials (ClinicalTrials.gov). However, MEK inhibitors are ineffective the most common malignancy associated with neurofibromatosis type 1: malignant peripheral nerve sheath tumors (MPNSTs) (8). Approximately $70 \%$ of MPNST patients present with unresectable and/or metastatic disease, and

Conflict of interest: The authors have declared that no conflict of interest exists. Submitted: October 20, 2015; Accepted: March 22, 2016.

Reference information: J Clin Invest. 2016;126(6):2181-2190. doi:10.1172/JCI85183. conventional chemotherapy and radiation do not improve overall survival; therefore, developing a treatment for these tumors represents a response to an urgent unmet clinical need (9).

In MPNSTs, mTORC1 is the critical PI3K pathway effector, and mTORC1 suppression alone exerts cytostatic effects in human cell lines and animal models $(5,10)$. While effects are only cytostatic, mTORC1 inhibitors are surprisingly more potent than MEK inhibitors, underscoring the importance of this pathway in this tumor type (11). Moreover, when combined with MEK inhibitors, mTORC1 inhibitors trigger cell death and dramatic tumor regression in vivo (11). Nevertheless, because these 2 major pathways regulate so many fundamental biological processes, it may be challenging to identify an effective therapeutic window in humans using combined MEK/ mTORC1 inhibitors. Therefore, we have been working to identify new therapeutic targets within these pathways that, when suppressed, may selectively affect cancer cells and spare normal tissue.

In the course of the studies presented here, we found that eIF4E, the limiting component of the eIF4F translation initiation complex, is a critical mTORC1 effector in MPNSTs. mTORC1 regulates eIF4E by phosphorylating and dissociating inhibitory eIF4E-binding proteins (4EBPs); however, eIF4E function is also controlled by phosphorylation at serine 209, which is exclusively mediated by the MAPK-interacting kinases (MNKs) 1 and 2 (12-14). MNK phosphorylation of eIF4E has been shown to enhance the translation of several prooncogenic mRNAs, and several mechanisms by which this may occur have 
been proposed (reviewed in ref. 13). Interestingly, studies have shown that while MNK kinases and eIF4E phosphorylation are dispensable for normal development and function, eIF $4 \mathrm{E}^{\mathrm{S} 209}$ phosphorylation is uniquely enhanced in several cancers and contributes to the development of prostate cancer and lymphoma (15-18). These findings demonstrate that the MNK/eIF4E signaling axis plays an important positive role in tumor development. Nevertheless, it is currently unknown whether the MNK kinases are actually therapeutic targets and are required for the survival of existing tumors. We reasoned that MPNSTs represent a unique setting for evaluating the therapeutic potential of MNK inhibition, as the MNK/eIF4E signaling axis might provide an alternative targetable node in these tumors, obviating the need for broad mTORC1 suppression.

Using genetic and chemical approaches, we show that MNK kinases are important therapeutic targets in MPNSTs and that combined MNK and MEK suppression represents a promising new therapeutic strategy for treating these currently incurable malignancies. Given that MNK kinases appear to be largely dispensable for normal cellular processes, a therapeutic index might be more readily achieved using combined MNK and MEK inhibitors. These studies also suggest that MNK inhibitors may represent an alternative to mTOR inhibitors in some settings.

\section{Results}

Combined eIF4E and MEK suppression kills MPNSTs. We previously showed that mTORC1 is critical for the proliferative and tumorigenic properties of NF1-mutant MPNSTs $(5,10)$. Moreover, when mTORC1 inhibitors are combined with MEK inhibitors, human MPNST cells die and tumors regress in a wellcharacterized genetically engineered MPNST model (Figure $1 \mathrm{~A}$ and ref. 11). Because eIF4E has been shown to be a critical downstream effector of mTORC1, we investigated whether suppression of eIF4E was sufficient to recapitulate the effects of mTORC1 inhibition in this tumor type. Notably, RNAi-mediated suppression of eIF4E alone exerted cytostatic effects and inhibited both proliferation and growth in soft agar in human (Figure $1, \mathrm{~B}-\mathrm{D})$ and mouse (Figure 1E) MPNST cell lines, thus mimicking the effects of mTOR inhibitors as single agents (Figure $1 \mathrm{~A}$ and ref. 11). However, when combined with the MEK inhibitor PD-0325901 (PD901), eIF4E suppression triggered cell death (Figure 1, F and G). These results demonstrate that eIF4E is a critical effector in NF1-mutant MPNSTs and that eIF4E suppression can cooperate with MEK inhibitors and substitute for mTORC1 suppression in this therapeutic setting.

The MNK/eIF4E signaling axis is activated in human and mouse MPNSTs. While mTORC1 activates eIF4E by phosphorylating and dissociating inhibitory $4 \mathrm{EBP}$ proteins, eIF4E function is also enhanced by phosphorylation at serine 209, which is exclusively regulated by MNK1 and MNK2 (reviewed in ref. 13). To determine whether MNK/eIF4E signaling was activated in MPNSTs, we evaluated the phosphorylation status of eIF4E at serine 209 in human and mouse MPNSTs. Immunoblots using a phosphospecific antibody demonstrated that eIF4E is hyperphosphorylated at serine 209 in human and mouse MPNST cells compared with normal cells (Figure 2A). Analysis of primary human and mouse tumor tissue further demonstrated that
eIF4E was phosphorylated in 9 of 10 and 4 of 5 tumors, respectively (Figure 2, B and C). These observations suggest that the MNK/eIF4E signaling axis is activated in a high percentage of MPNSTs, warranting further investigation of the therapeutic potential of targeting this pathway.

Genetic suppression of MNK kinases cooperates with MEK inhibitors to promote MPNST cell death. To evaluate the potential therapeutic effects of MNK inhibition, MNK2 and MNK1 were knocked down both independently and in combination. Suppression of either MNK2 or MNK1 alone led to a substantial but incomplete decrease in eIF4E phosphorylation that was completely lost when MNK1 and MNK2 were concomitantly suppressed, indicating that both highly related kinases contribute to eIF4E phosphorylation in these tumors (Figure 2D). We next examined the biological consequences of MNK suppression in the presence and absence of MEK inhibitors. Genetic ablation of either MNK1 or MNK2 alone slightly inhibited proliferation, but killed cells when combined with PD901 (Figure 2E). Concomitant suppression of MNK1 and MNK2 further enhanced this cytotoxic response (Figure 2E). These results demonstrate that the combined suppression of MNK and MEK kinases potently kills MPNSTs, revealing potential therapeutic strategies for these incurable malignancies.

Therapeutic agents that suppress MNK kinases cooperate with MEK inhibitors. To determine whether chemical inhibition of MNK kinases could recapitulate the effects of genetic suppression, we first utilized the MNK1 and MNK2 inhibitor CGP57380 (19). Similar to what occurs with genetic ablation of MNK1 and MNK2, CGP57380 inhibited eIF4E $\mathrm{E}^{\text {S209 }}$ phosphorylation in human MPNST cells (Figure 3A) and, on its own, partially suppressed proliferation (Figure 3B). Moreover, cells treated with a combination of CGP57380 and PD901 died (Figure 3B). Cercosporamide, a natural product that also inhibits MNK kinases (20), also suppressed eIF4 $\mathrm{E}^{\mathrm{S} 209}$ phosphorylation (Figure 3C) and killed MPNST cells in a dose-dependent fashion when combined with PD901 (Figure 3D). Because CGP57380 and cercosporamide are both tool compounds that are not being clinically developed, we investigated whether any other existing kinase inhibitors might suppress MNK and therefore could be more readily evaluated in vivo. Interestingly, the multikinase inhibitor merestinib/LY2801653, originally designed to suppress the receptor tyrosine kinase MET, has been shown to directly inhibit MNK1 and MNK2 kinases (21). Similarly, we found that the FDA-approved compound cabozantinib, a second MET/multikinase inhibitor, also directly bound MNK1 and MNK2 with a Kd of $790 \mathrm{nM}$ and $21 \mathrm{nM}$, respectively (Figure 3E), and suppressed eIF4E $\mathrm{E}^{\mathrm{S} 209}$ phosphorylation in MPNSTs at even lower concentrations than CGP57380 (Figure 3F). Moreover, cabozantinib exerted a dose-dependent effect on eIF $4 E^{\mathrm{S} 209}$ phosphorylation and MPNST cell death when combined with PD901 (Figure 3, G and H).

The therapeutic effects of cabozantinib/PD901 require the suppression of MNK-induced eIF $4 E^{S 209}$ phosphorylation. While these studies demonstrate that cabozantinib effectively inhibits MNK kinases, it was originally developed as an MET kinase inhibitor (22). Therefore, to independently examine the effects of MET suppression in MPNSTs, we evaluated the effects of the structurally unrelated selective MET kinase inhibitor PF-04217903, 
A
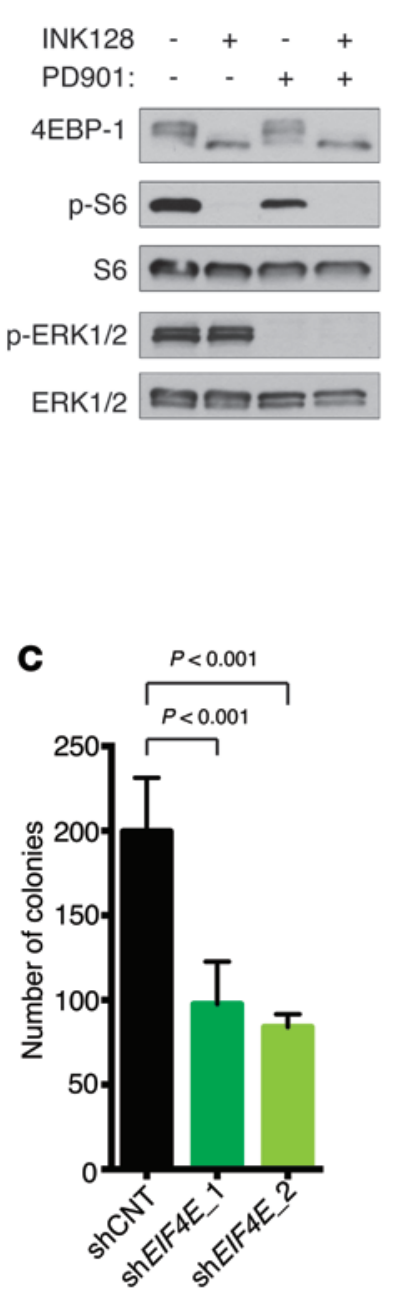
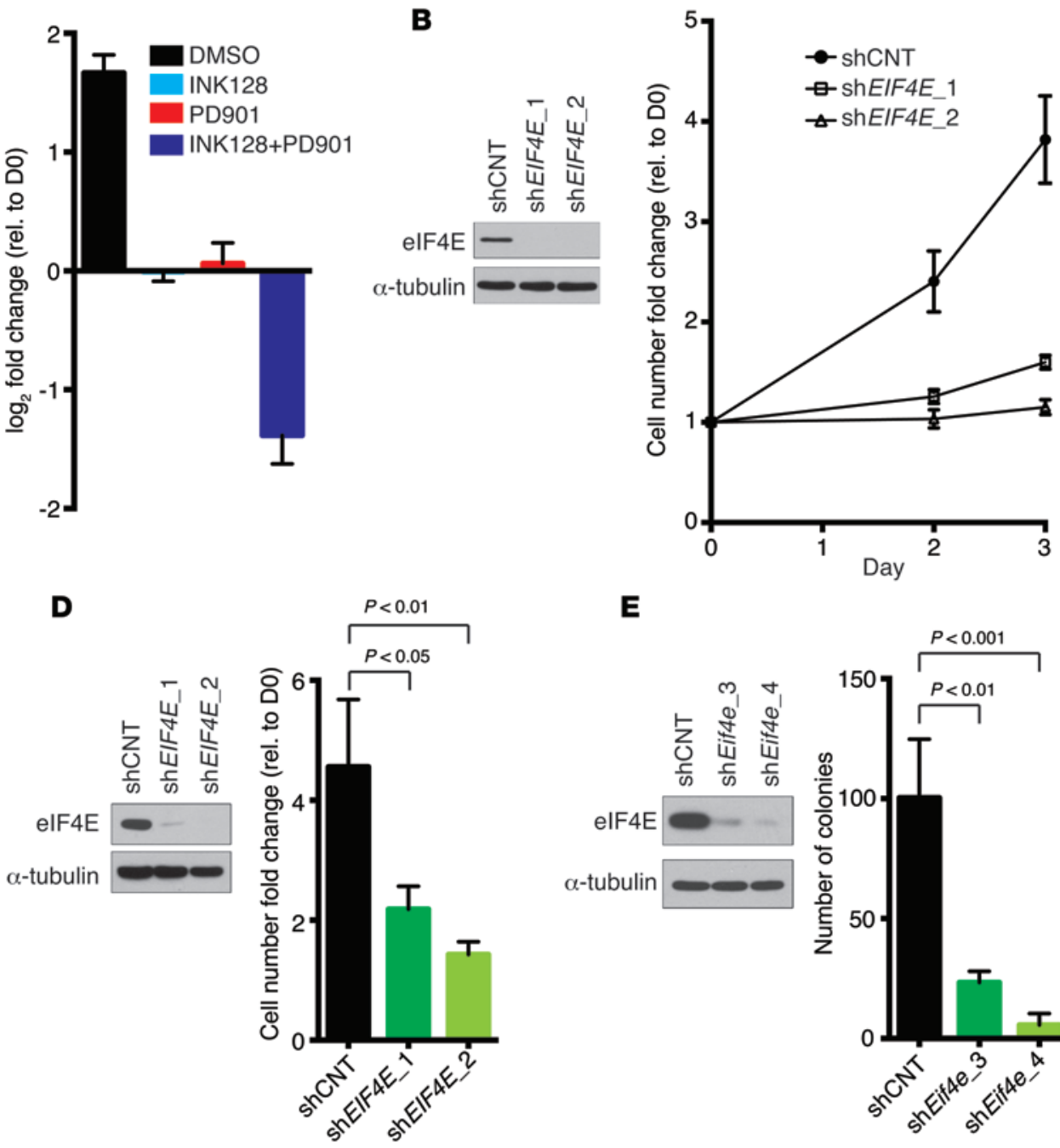

E

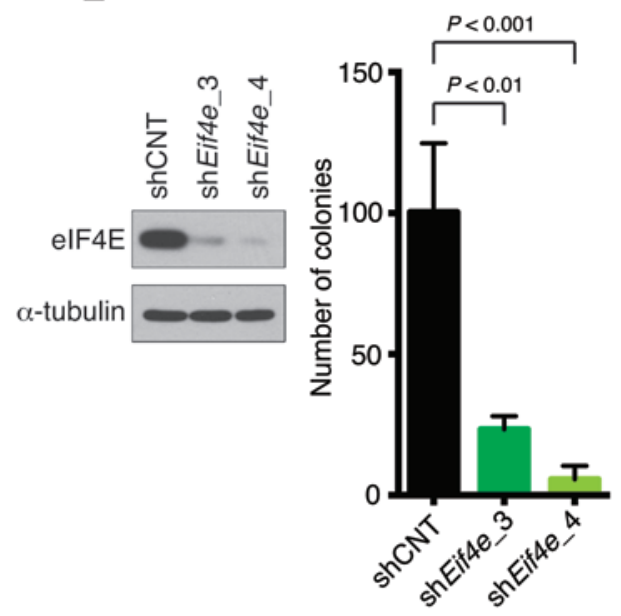

$\mathbf{F}$

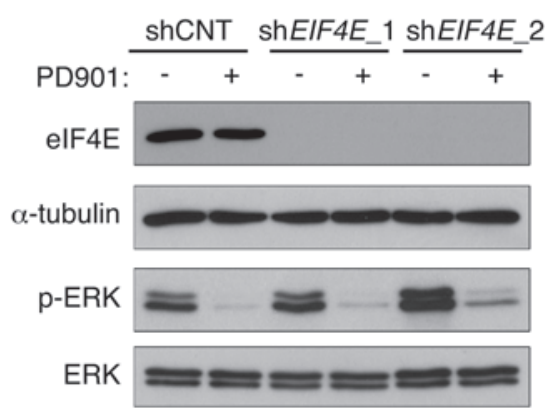



Figure 1. elF4E is a critical mTORC1 effector in MPNSTs. (A) (Left) Immunoblots of p-ERK, 4EBP1, and p-S6 in S462 treated with 200 nM INK128 (an mTOR kinase inhibitor) and $750 \mathrm{nM}$ PD901 (a MEK inhibitor) for 24 hours. (Right) Change in cell number of S462 cells treated with above concentrations of INK128 or PD901 alone or in combination. Graph represents the average $\log _{2}$ of fold change in cell number 72 hours after treatment relative to time 0 (mean $\pm S D, n=3$ ). Note that -1 on the $y$ axis corresponds to a 50\% decrease in cell number. (B) Immunoblot of elF4E (left) and fold change in cell number on days 2 and 3 relative to day 0 (right) and of S462 cells stably expressing 2 unique shRNAs against EIF4E (shEIF4E_1 and shEIF4E_2) or shCNT (mean \pm SD, $n=3$ ). (C) Number of colonies formed in soft agar by 5462 cells expressing shCNT, shEIF4E_1, or shEIF4E_2 (mean \pm SD, $n=4$, 1-way ANOVA followed by Bonferroni's multiple comparisons test). (D) elF4E levels (left) and fold change in cell number after 72 hours (right) of 90-8TL cells expressing shEIF4E_1, shEIF4E_2, or shCNT (mean \pm SD, $n=3$, 1-way ANOVA followed by Bonferroni's multiple comparisons test). (E) Number of soft agar colonies formed by CM173 cells (mouse MPNST) expressing shCNT or 2 independent shRNAs against Eif4e (shEif4e_3 and shEif4e_4) (mean \pm SD, $n=3$, 1-way ANOVA followed by Bonferroni's multiple comparisons test). (F) Levels of elF4E and p-ERK in S462 cells stably expressing shCNT, shEIF4E_1, or shEIF4E_2 treated with 750 nM PD901 for 24 hours. (C) Change in cell

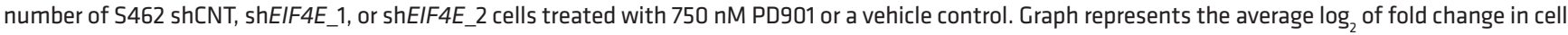
number 72 hours after treatment with PD901 relative to time 0 (mean $\pm S D, n=3$ ). Experiments were conducted at least 3 times for validation. 
A

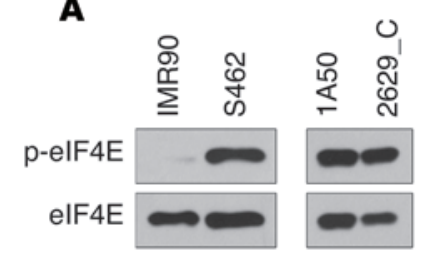

D

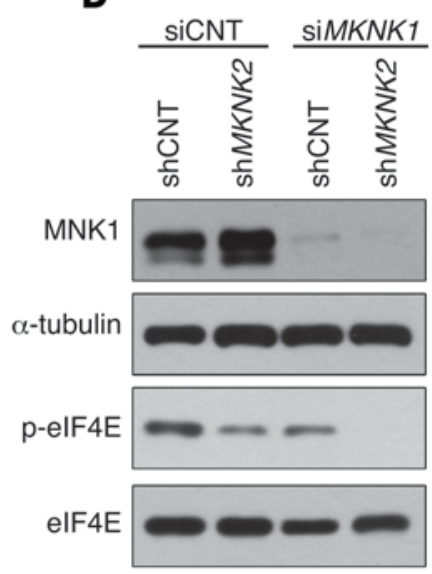

B

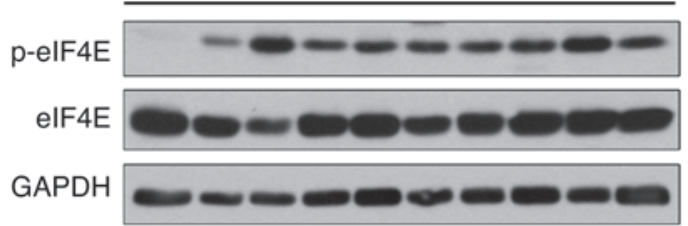

C

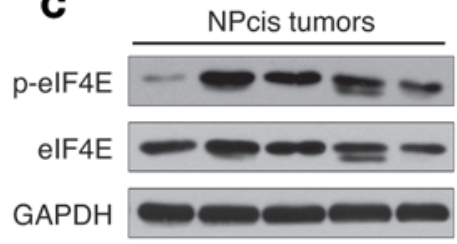

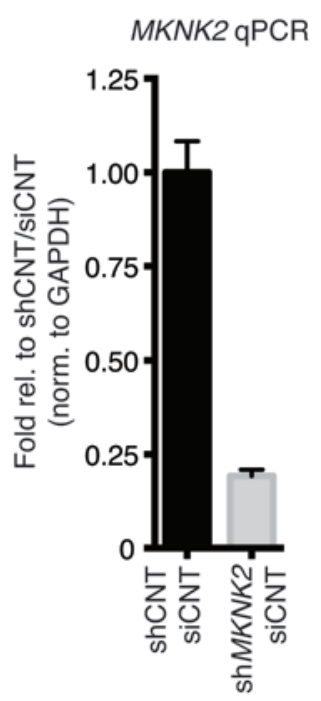

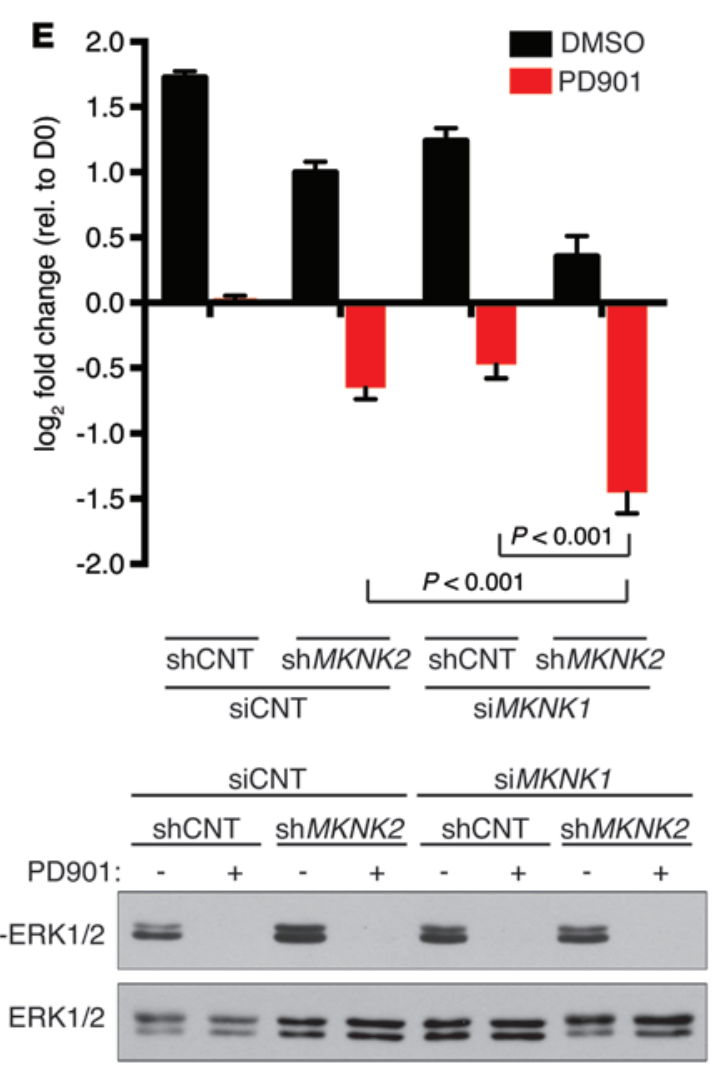

Figure 2. MNK kinases are frequently activated in MPNSTs, and genetic ablation triggers cell death when combined with MEK inhibitors. (A) (Left) Immunoblot using a phospho-specific (S209) elF4E antibody of lysates from normal human fibroblasts (IMR90) and MPNST cells (S462) and (Right) mouse MPNST cell lines (1A50 and 2629_C). (B) elF4E phosphorylation levels in lysates from primary human MPNSTs. (C) Levels of elF4E phosphorylation in primary mouse MPNSTs. (D) (Left) MNK1 and p-elF4E levels following expression of shMKNK2, transfection with siRNA against MKNK1 (siMKNK1), or combined shMKNK2 expression and siMKNK1 transfection in 5462 cells. (Right) Because existing MNK2 antibodies are not specific, mRNA levels of MKNK2 in shMKNK2-expressing S462 cells are shown relative to nontargeting controls and normalized to GAPDH (mean \pm SD, $n=3$ ). (E) (Top) Change in cell number of S462 expressing shCNT or shMKNK2 transfected with siMKNK1 or siCNT and treated with $750 \mathrm{nM}$ PD901 or a vehicle control (DMSO). Graph represents the average $\log _{2}$ of fold change in cell number 72 hours after treatment with PD901 relative to time 0 (mean \pm SD, $n=3,1$-way ANOVA followed by Bonferroni's multiple comparisons test). (Bottom) Levels of p-ERK in the corresponding cell lines following 24 hours of treatment with $750 \mathrm{nM}$ PD901. Experiments repeated at least 3 times for validation.

which did not suppress eIF4E phosphorylation (Figure 4A and ref. 23). PF-04217903 effectively suppressed MET phosphorylation in MPNST cells, but did not significantly affect proliferation (Figure 4, A and B). Moreover, combined PF-04217903/PD901 did not trigger cell death or promote further proliferative suppression compared with PD901 alone (Figure 4B). Other major targets of cabozantinib include VEGFR2, AXL, and c-KIT kinases. Immunoblot analysis revealed that c-KIT is not expressed in these MPNST cells (data not shown). We therefore investigated whether suppression of AXL or VEGFR2 might be mediating the observed cytotoxicity. Unlike MNK suppression, which was sufficient to kill MPNST cells in the presence of PD901 (Figure 2E), genetic ablation of AXL or VEGFR2 had no effect on the survival or proliferation of cells alone or when combined with PD901 (Figure 4, C and D). Importantly, as observed with MET suppression, inhibition of these cabozantinib targets also had no effect on eIF4E phosphorylation (Figure 4, A and C), indicating cabozantinib-mediated suppression of eIF4E phosphorylation occurs solely through direct MNK inhibition as opposed to downregulation of upstream kinases that might converge on MNK1 and MNK2. Collectively, these genetic and chemical studies demonstrate that (a) MNK suppression is sufficient to cooperate with MEK inhibitors to kill NF1-mutant MPNSTs, (b) several structurally unrelated agents that directly suppress MNK kinase activity 


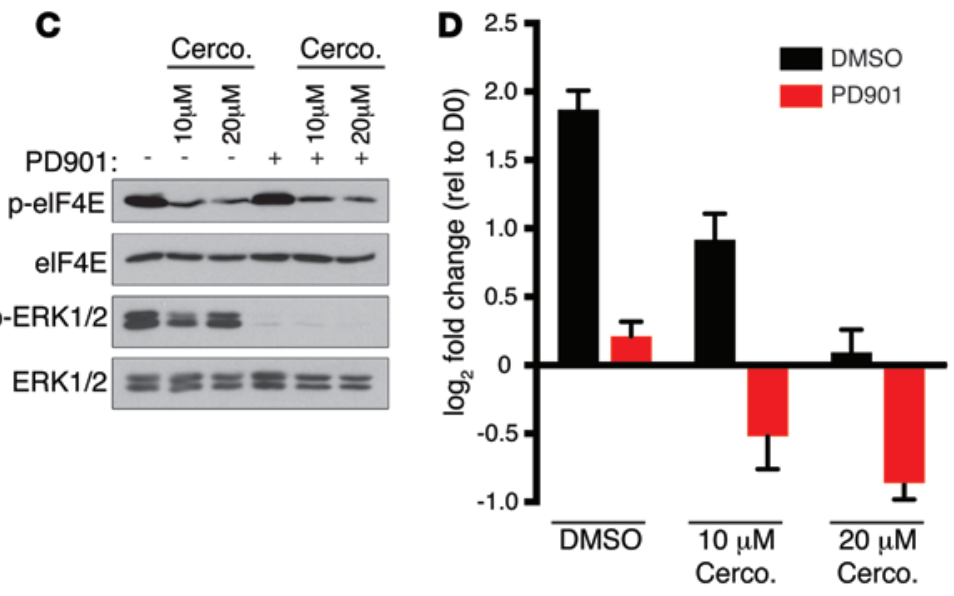
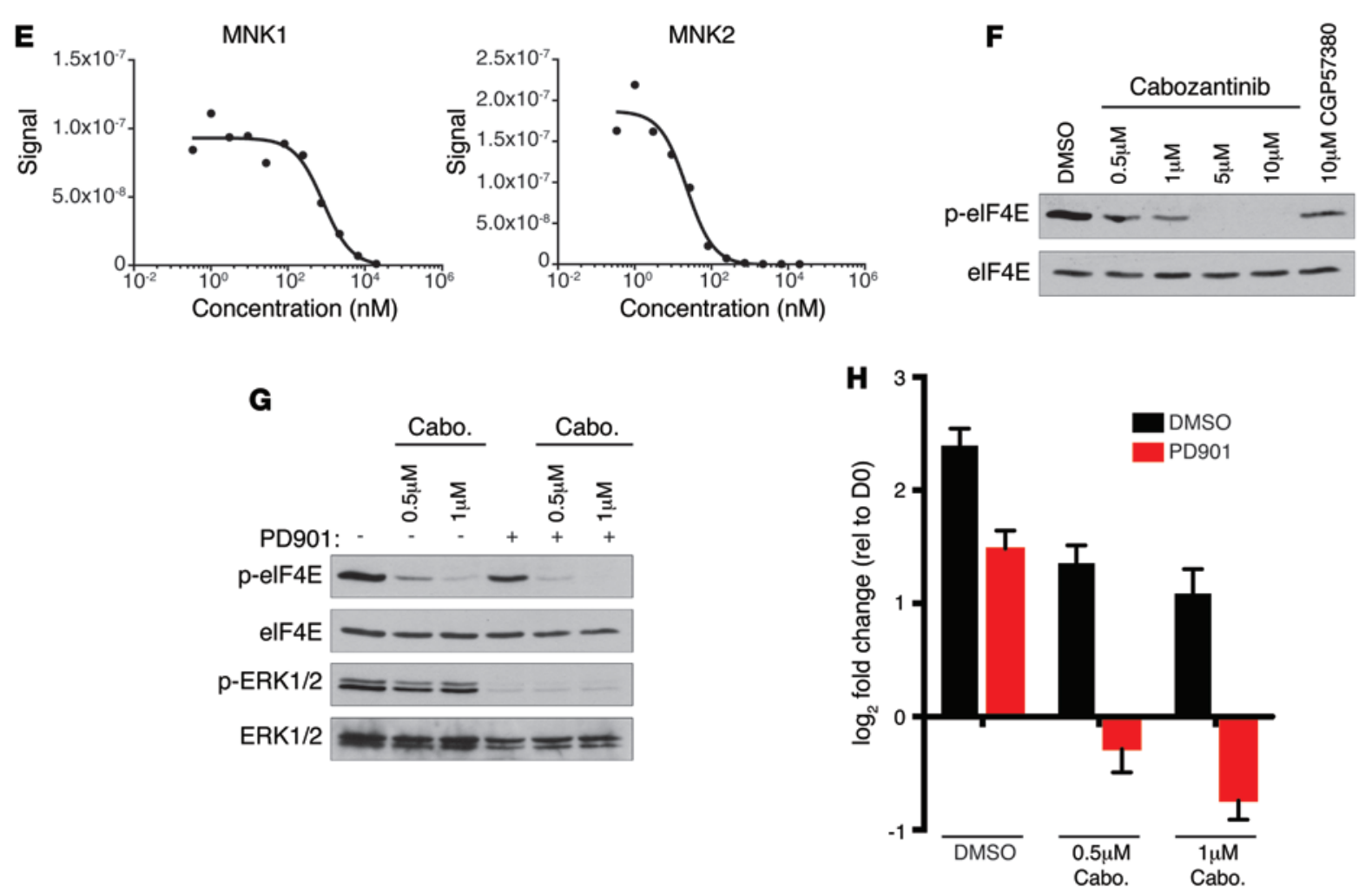

Figure 3. Multiple therapeutic agents that suppress MNK kinases cooperate with MEK inhibitors. (A) p-elF4E and p-ERK1/2 levels in S462 cells treated with DMSO, $10 \mu \mathrm{M}$ CGP57380, 750 nM PD901, or both inhibitors for 24 hours. (B) Change in cell number of S462 cells treated with $10 \mu \mathrm{M}$ CGP57380 (CCP) or $750 \mathrm{nM} \mathrm{PD901} \mathrm{alone} \mathrm{or} \mathrm{in} \mathrm{combination.} \mathrm{Graph} \mathrm{represents} \mathrm{the} \mathrm{average} \log _{2}$ of fold change in cell number 72 hours after treatment relative to time 0 (mean $\pm S D, n=3$ ). (C) p-elF4E and $p$-ERK1/2 levels in 5462 cells treated with indicated concentrations of cercosporamide (Cerco.) alone or in combination with $750 \mathrm{nM}$ PD901 for 24 hours. (D) Change in cell number of S462 cells treated with $10 \mu \mathrm{M}$ or $20 \mu \mathrm{M}$ cercosporamide or 750 $\mathrm{nM}$ PD901 alone or in combination. Graph represents the average $\log _{2}$ of fold change in cell number 72 hours after treatment relative to time 0 (mean $\pm \mathrm{SD}, n=3$ ). (E) Binding of tagged MNK1 and MNK2 to immobilized ligand was measured in the presence of increasing cabozantinib. Tagged MNK kinases were quantified by real-time qPCR using previously described methods (26). Representative curves are shown. (F) Levels of p-elF4E following 24 hours of treatment with increasing concentrations of cabozantinib or $10 \mu \mathrm{M}$ CGP57380. (G) $p$-elF4E and p-ERK1/2 levels in S462 cells treated with $0.5 \mu \mathrm{M}$ or $1 \mu \mathrm{M}$ cabozantinib (Cabo) alone or in combination with $750 \mathrm{nM}$ PD901 for 24 hours. (H) Change in cell number of S462 treated with the indicated concentrations of cabozantinib or $750 \mathrm{nM}$ PD901 alone or in combination. Graph represents the average log of fold change in cell number 72 hours after treatment relative to time 0 (mean $\pm S D, n=3$ ). Experiments repeated at least 3 times for validation.

similarly cooperate with MEK inhibitors to kill these tumor cells, and (c) MET, AXL, and VEGFR2 suppression are not required nor sufficient to mediate the cytotoxic effects.

We initially began investigating MNK kinases as potential therapeutic targets in MPNSTs because they uniquely phosphor- ylate eIF $4 \mathrm{E}$ at S209 and because eIF $4 \mathrm{E}$ is such a critical mTOR effector in these tumor cells. To confirm that the inhibition of eIF $4 \mathrm{E}^{\mathrm{S} 209}$ phosphorylation was contributing to the therapeutic effects, we generated a previously described phosphomimetic mutant in which the serine at position 209 is substituted by an 
A

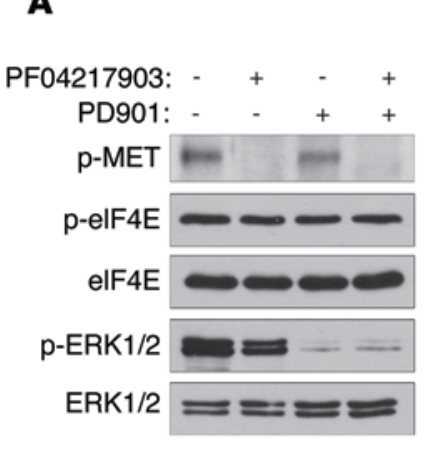

B

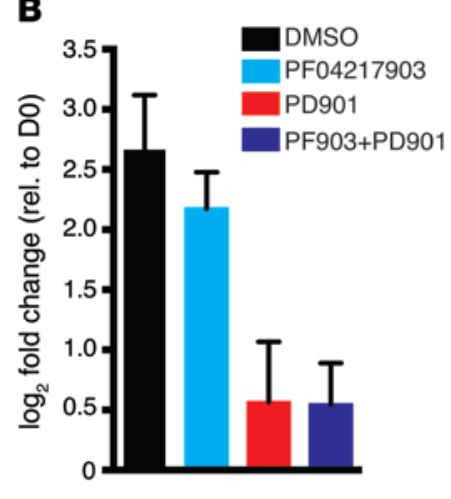



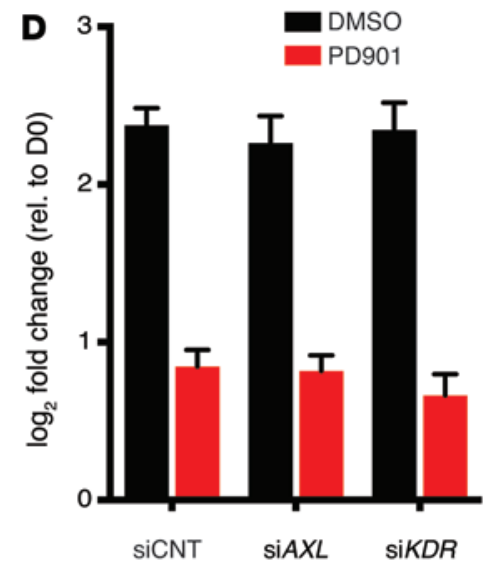

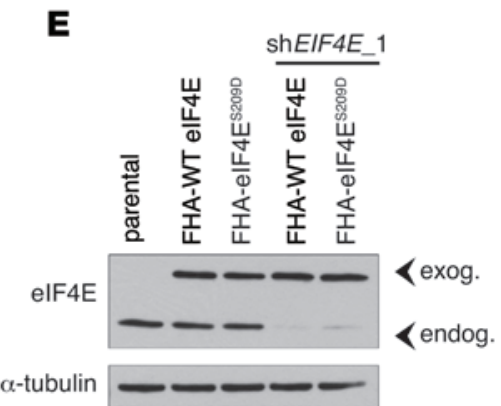
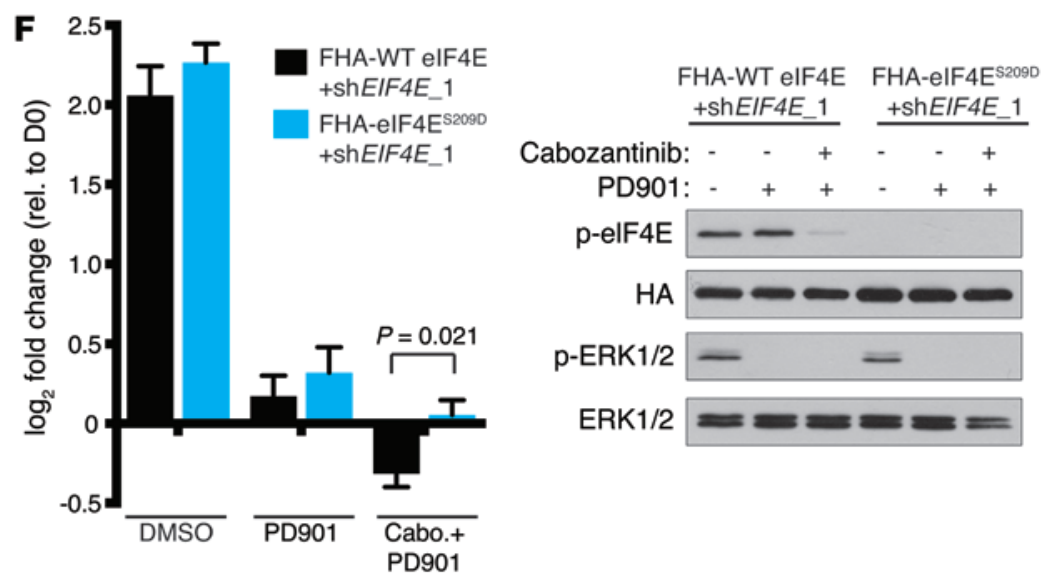

G
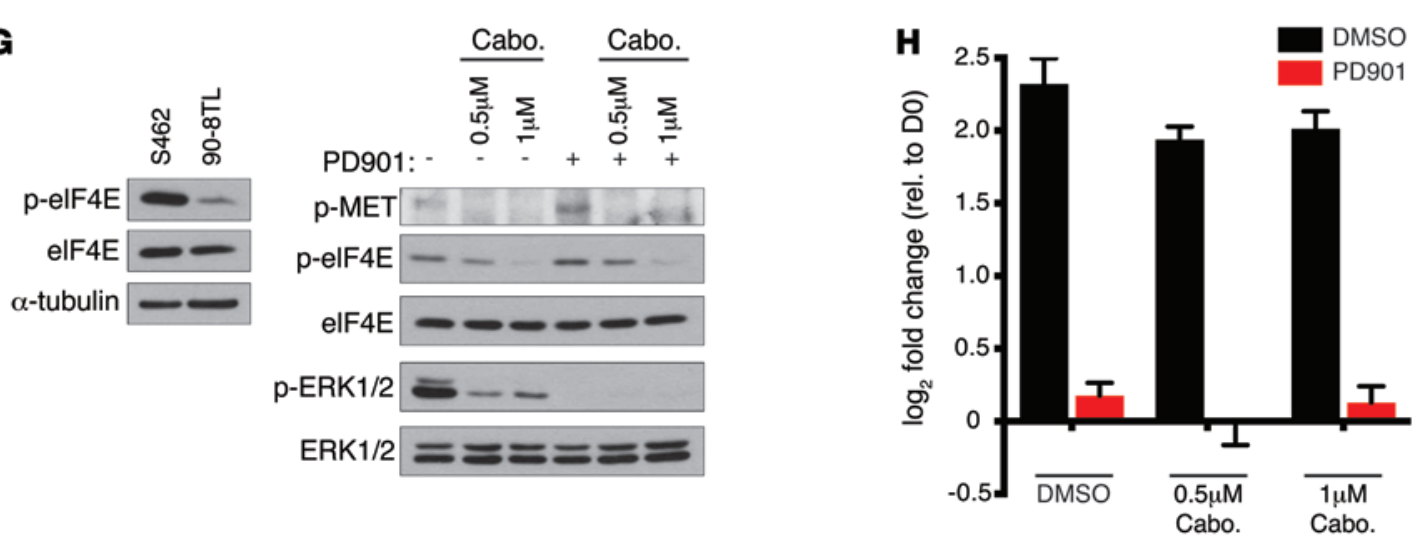

Figure 4. Cabozantinib cooperates with PD901 by suppressing MNK and elF4E phosphorylation. (A) $p$-MET, p-elF4E, and p-ERK1/2 levels in S462 cells 24 hours after treatment with $1 \mu \mathrm{M}$ PF04217903 and $750 \mathrm{nM}$ PD901. (B) Change in cell number after treatment with $1 \mu \mathrm{M}$ PF04217903 (PF903) and/or 750 nM PD901. Graph represents the average $\log _{2}$ of fold change in cell number 72 hours after treatment relative to time 0 (mean \pm SD, $n=3$ ). (C) AxI, VEGFR-2, p-elF4E, and p-ERK1/2 levels in S462s following transfection with siAXL, siKDR, or control siRNA (siCNT). (D) Change in cell number following transfection with si $A X L$, siKDR, or siCNT and treatment with $750 \mathrm{nM}$ PD901. Graph represents the average $\log _{2}$ of fold change in cell number 72 hours after treatment relative to time 0 (mean $\pm S D, n=3$ ). (E) elF4E levels in parental S462 cells or cells expressing exogenous Flag-HA-tagged (FHA) elF4E WT or elF4E ${ }^{\text {S2090 }}$ coexpressing shEIF4E_1 against endogenous elF4E. (F) (Left) Change in cell number of FHA-WT elF4E- and FHA-elF4E ${ }^{52090}$-expressing cells coexpressing shEIF4E_1 treated with $750 \mathrm{nM}$ PD901 alone or in combination with $0.5 \mu \mathrm{M}$ cabozantinib. Graph represents the average log of $_{2}$ fold change in cell number 72 hours after treatment relative to time 0 (mean $\pm S D, n=3, t$ test). (Right) $p$-elF4E and $p$-ERK1/2 levels in cells described above 24 hours after treatment. (C) (Left) Levels of p-elF4E in 90-8TL human MPNST cells relative to S462 cells. (Right) p-MET, p-elF4E, and p-ERK1/2 levels in 90-8TL cells treated with $0.5 \mu \mathrm{M}$ or $1 \mu \mathrm{M}$ cabozantinib alone or combined with $1 \mu \mathrm{M}$ PD901. (H) Change in cell number of 90-8TL cells treated with the indicated concentrations of cabozantinib or $1 \mu \mathrm{M}$ PD901 alone or in combination. Graph represents the average $\log _{2}$ of fold change in cell number 72 hours after treatment relative to time 0 (mean $\pm S D, n=3$ ). Experiments repeated at least 3 times for validation. 
A

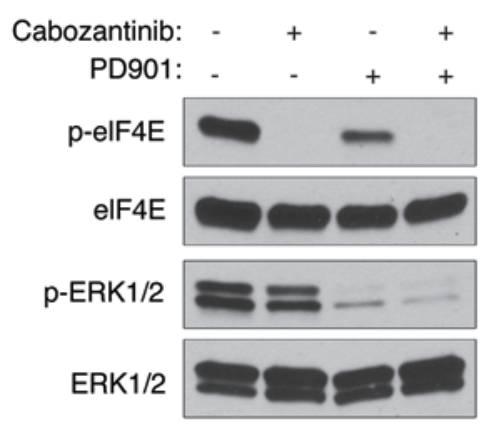

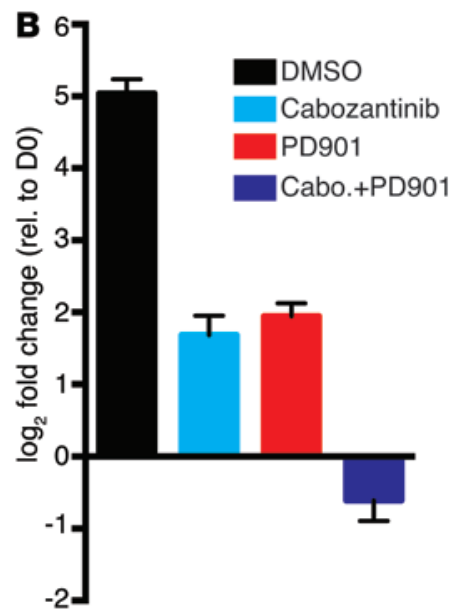

C

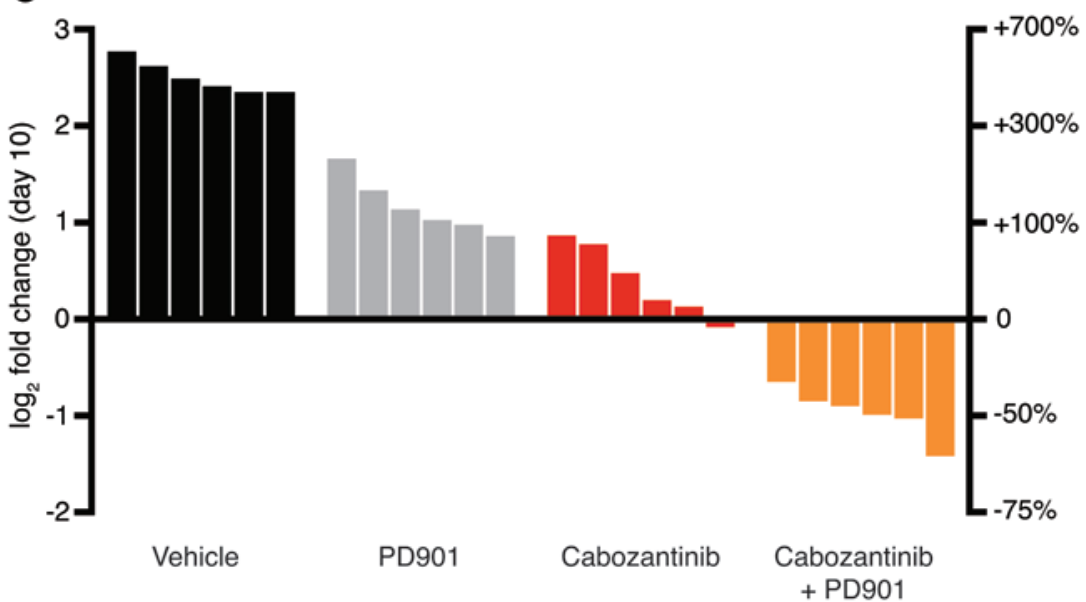

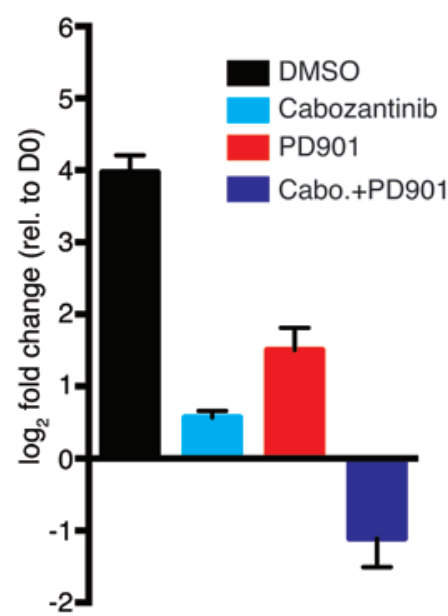

D

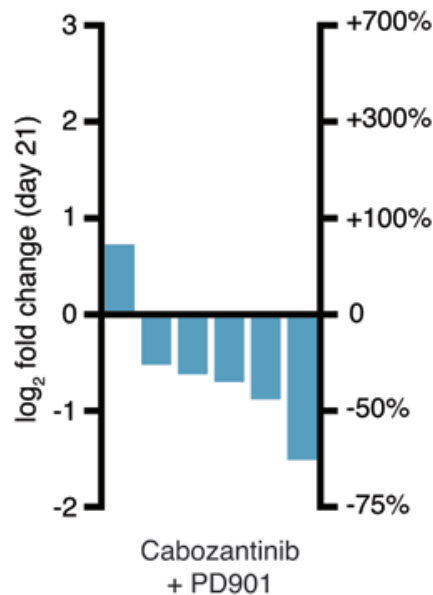

Figure 5. Combined cabozantinib and PD901 treatment induces tumor regression in vivo. (A) Levels of $p$-elF4E and p-ERK1/2 in CM261 cells (mouse MPNST line) treated with $5 \mu \mathrm{M}$ cabozantinib and $2 \mu \mathrm{M}$ PD901 alone or in combination. (B) Graph depicting the change in cell number of mouse (CM261, left, and CM173, right) MPNST cells treated with $5 \mu \mathrm{M}$ cabozantinib or $2 \mu \mathrm{M}$ PD901 alone or in combination. Graph represents the average log of fold change in cell number 96 hours after treatment relative to time 0 (mean $\pm S D, n=3$ ). Experiments repeated at least 3 times for validation. (C) Waterfall plot depicting change in tumor volume after 10 days of treatment with cabozantinib at $12.3 \mathrm{mg} / \mathrm{kg} / \mathrm{d}$ (red bars) or in combination with PDg01 at $1.5 \mathrm{mg} / \mathrm{kg} / \mathrm{d}$ (orange bars) $(P=0.0022$, Mann-Whitney $U$ test, cabozantinib vs. cabozantinib + PD901), as compared with vehicle (black), and $1.5 \mathrm{mg} / \mathrm{kg} / \mathrm{d} P D 901$ alone (gray). Vehicle and PD901 tumor responses are previously published historic controls adapted with permission from Cancer Discovery (11). (D) Waterfall plot of the change in tumor volume of mice treated with $12.3 \mathrm{mg} / \mathrm{kg} / \mathrm{d}$ cabozantinib in combination with $1.5 \mathrm{mg} / \mathrm{kg} / \mathrm{d}$ PDg01 for 21 days. For both C and $\mathbf{D}$, each bar represents an individual tumor-bearing mouse. The left $y$ axis indicates the $\log _{2}$ of the fold change in volume over the indicated time relative to day 0 measurements. The right $y$ axis indicates the equivalent percentage change in tumor volume.

aspartic acid (eIF4E $\mathrm{E}^{\mathrm{S} 209 \mathrm{D}}$ ) (18). Flag-HA-tagged eIF4E $\mathrm{E}^{\mathrm{S} 209 \mathrm{D}}$ and WT eIF4E were further engineered with sequential silent mutations to prevent targeting by shEIF4E_1 and were ectopically expressed at physiological levels in MPNST cells together with shEIF4E_1 to suppress endogenous eIF4E expression (Figure $4 \mathrm{E})$. Notably, the cells expressing eIF $4 \mathrm{E}^{\mathrm{S} 209 \mathrm{D}}$ were significantly less sensitive to cabozantinib/PD901 compared with cells expressing WT eIF4E (Figure 4, E and F). These observations demonstrate that the suppression of MNK-dependent eIF4E phosphorylation contributes to MPNST cell death triggered by combined cabozantinib/MEK inhibitors. Interestingly, while 9 of 10 human and 4 of 5 mouse MPNSTs exhibited robust activation of the MNK/eIF4E signaling axis (Figure 2, B and C), we identified a human MPNST cell line that exhibited extremely low levels of eIF4E S209 phosphorylation (Figure 4G). While cabozantinib effectively suppressed MET phosphorylation in these cells, it had minimal effects on proliferation as a single agent and did not trigger cell death when combined with PD901 (Figure 4, G and H), consistent with the importance of the MNK/ eIF4E signaling axis in conferring sensitivity.

Combined cabozantinib/PD901 treatment promotes tumor regression in vivo. Finally, to test the efficacy of combined MNK and MEK inhibition in vivo, we evaluated the effects of cabozantinib and PD901 in a genetically engineered mouse MPNST model (24). Like human MPNSTs, tumors from these animals harbor compound mutations in Nf1 and Trp53. MPNSTs that develop in these animals are highly aggressive, and mice survive for an average of 10.7 days after tumors are detected, recapitulating the aggressive nature of human tumors (24). As shown in Figure 5A, eIF4E was phosphorylated on serine 209 in mouse 
MPNST cells and was potently inhibited in response to cabozantinib. Moreover, combined cabozantinib/PD901 treatment promoted cell death in mouse MPNST lines (Figure 5B). We therefore evaluated the therapeutic effects of cabozantinib/PD901 in vivo. Genetically engineered mice harboring Nf1/p53 mutant MPNSTs were treated with cabozantinib at $12.3 \mathrm{mg} / \mathrm{kg} / \mathrm{d}$ alone or in combination with $1.5 \mathrm{mg} / \mathrm{kg}$ PD901 daily. (Figure 5C). Similarly to in vitro observations, cabozantinib alone exerted cytostatic effects; however, when combined with PD901, every tumor regressed, shrinking between $36 \%$ and $63 \%$ (Figure 5C). We performed a second preclinical study, extending treatment for 21 days, and found that the tumor regression was maintained, which is particularly striking given that these tumors grow on average 4- to 7-fold and kill control mice in half of this time (Figure 5D). Together with the genetic analysis provided, these findings suggest that combined MEK/MNK inhibition represents a promising therapeutic strategy for treating NF1mutant MPNSTs. While, cabozantinib or other multikinase inhibitors that suppress MNK kinases could be evaluated in this therapeutic context in clinical trials, more importantly, these findings support the potential utility of selective MNK inhibitors for these and likely other cancers.

\section{Discussion}

MPNSTs are highly aggressive malignancies that develop in neurofibromatosis type 1 patients or can arise spontaneously (8). These tumors frequently metastasize, and there are currently no effective treatments for unresectable lesions, which are lethal in $70 \%$ of cases (9). Here, we identify what we believe is a new therapeutic target in MPNSTs and provide a promising therapeutic strategy for these deadly malignancies. Using a variety of genetic and chemical approaches, we show that suppression of MNK kinases effectively cooperates with MEK inhibitors to kill human MPNST cells. Moreover, we find that the multikinase inhibitor cabozantinib directly inhibits MNK1 and MNK2 and cooperates with MEK inhibitors to induce dramatic MPNST regression in vivo. Importantly, these therapeutic effects cannot be recapitulated by suppressing other cabozantinib targets and require the suppression of MNK-induced eIF4E phosphorylation. Together, these studies highlight the potential therapeutic utility of MNK kinase inhibitors in cancer and provide an important clinical setting in which they should be evaluated. Notably, at least 2 phase I studies of more selective MNK inhibitors are currently underway (ClinicalTrials.gov eFT508, BAY 1143269).

Previous studies have shown that MNK is important for the development of PTEN-mutant cancers and that loss-offunction mutations delay tumor formation in mouse models (17). However, not all genes that contribute to tumor development are therapeutic targets. Here, we show that acute suppression of MNK plays a causal role in mediating an effective therapeutic response when combined with MEK inhibitors. Moreover, we find that $\mathrm{MNK}$ is an unappreciated direct target of the FDA-approved drug cabozantinib, suggesting that trials evaluating the combined effects of cabozantinib and MEK inhibitors should be considered. Notably, while we have found that a relatively low preclinical dose of cabozantinib is effective in this model when combined with a dose of PD901 that is readily achieved in patients, the potential combined toxicity of these agents in humans still needs to be taken into account. However, the more important finding in this study is that selective MNK kinase inhibitors should be effective in NF1-mutant tumors, which would circumvent any toxicity associated with inhibiting other cabozantinib targets. Moreover, because MNK appears to be a fairly cancer-specific target, MNK inhibitors may have a unique therapeutic window. The discovery that MNK is the functional therapeutic target of cabozantinib in these tumors may be reminiscent of the finding that anaplastic lymphoma receptor tyrosine kinase (ALK) is the key target of the multikinase inhibitor crizotinib in lung cancer (25). Notably, the initial ALK/crizotinib discovery led to the development of more selective ALK inhibitors that appear to be more effective than crizotinib and can overcome crizotinib resistance (25). The recent emergence of more MNK-selective inhibitors may provide a unique opportunity for beginning clinical studies with these more specific agents from the onset. Finally, while these studies validate MNK as an important new therapeutic target in MPNSTs, this combination could also be examined in other Ras and/or mTOR-dependent cancers.

eIF4E is a known MNK target, and our phosphomimetic studies demonstrate that eIF4E dephosphorylation is an important mediator of the therapeutic response. However, MNK kinases have additional known and likely unknown substrates (reviewed in ref. 13). Some of these can be conceptually ruled out as contributors to the therapeutic response, such as Sprouty2, which would be expected to be destabilized by MNK inhibition (reviewed in ref. 13). While it will be interesting to elucidate the complete therapeutic effects of MNK suppression, our studies suggest that eIF4E phosphorylation at serine 209 can serve as a useful biomarker to identify tumors in which MNK kinases are activated. Importantly, we find that MNK kinases are activated in a high percentage of primary human MPNSTs. A relatively homogenous activation of this pathway might be expected in tumors from patients with a familial cancer syndrome that overall exhibits less heterogeneity than sporadic cancers. However, eIF4E phosphorylation can be used to identify other tumor types or tumor subsets that might benefit from this therapeutic approach. Taken together, these studies demonstrate that combined suppression of MNK and MEK potently kills MPNSTs and have established a therapeutic paradigm for these untreatable malignancies.

\section{Methods}

Cell lines and reagents. S462s and IMR90s were purchased from ATCC. The 90-8TLs were provided by Eric Legius (Catholic University Leuven). CM261, CM173, 1A50, and 2629_C were generated from MPNSTs isolated from NPcis mice. All cell lines were maintained in DMEM supplemented with $10 \%$ FBS, penicillin/ streptomycin, and L-glutamine. Antibodies were obtained from the following sources: Cell Signaling Technologies: eIF4E (catalog 9742), MNK1 (C4C1) (catalog 2195), p-Met (D26) (catalog 077), p-ERK1/2 (D13.14.4E) (catalog 4370), ERK1/2 (catalog 9102), pS6 (catalog 2215), S6 (5G10) (catalog 2217), GAPDH (14C10) (catalog 2118), 4E-BP1 (catalog 9452), and VEGFR2 (55B11) (catalog 2479); Invitrogen/Life Technologies: p-eIF4E (catalog 44528G); SigmaAldrich: $\alpha$-tubulin (B-5-1-2) (catalog T5168); R\&D Systems: Axl 
(catalog AF154); and Covance: HA.11 (MMS-101P). INK128 was purchased from Active Biochem. CGP57380 and cercosporamide were purchased from Tocris Bioscience. PF04217903 was purchased from Selleck Chemicals. Cabozantinib was purchased from ChemieTek. PD901 was a gift from Kevin Shannon (UCSF, San Francisco, California, USA).

Human MPNST samples. All patients were diagnosed as having neurofibromatosis type 1 .

RNAi. pLKO.1 lentiviral expression plasmids containing shRNAs against eIF4E (human and mouse) and MNK2 were obtained from the Broad RNAi Consortium. The target sequences for hairpins directed against human eIF4E were as follows: shEIF4E_1 (TRCNO000062574): CCAAAGATAGTGATTGGTTAT; and shEIF4E_2 (TRCNO000299576): CCGACTACAGAAGAGGAGAAA. The target sequences for hairpins directed against mouse eIF4E were as follows: shEIF4E_3 (TRCNO000077475): CCGAAGATAGTGATTGGTTAT; and shEIF4E_4 (TRCNO000077477): CGATTGATCTCTAAGTTTGAT. The target sequence for hairpins directed against human MNK2 is shMNK2 (TRCNO000342285): CGCCGTCAAGATCATTGAGAA. Nontargeting, siMNK1, siAXL, and siKDR pools were purchased from Dharmacon (D-001810-10, L-004879-00, L-003104-00, and L-003148-00, respectively). siRNAs were transfected into cells using RNAiMax lipofectamine reagent from Invitrogen.

Soft agar, cellular proliferation, and cell death studies. For soft agar experiments, 50,000 S462 cells or 25,000 CM173 cells were suspended in $0.35 \%$ agar in growth media on top of $0.5 \%$ agar in growth media in 6-well plates. After 3 weeks, colonies were fixed and stained with crystal violet; plates were scanned and the number of colonies per well was determined using ImageJ software (NIH). For cellular proliferation and cell death assays, 150,000 cells per well were seeded in 6-well plates, and approximately 24 hours after plating, day 0 counts were taken. For inhibitor experiments, drug treatments were started on day 0 , and for siRNA experiments, cells were transfected approximately 24 hours before plating. All S462 cell death assays were performed in DMEM with $2 \%$ FBS starting on day 0 . Final cell counts were taken at 72 or 96 hours where indicated to determine changes in cell number from day 0. For Western blots to determine drug efficacy or efficient knockdown, lysates were collected 24 hours following the addition of inhibitors.

eIF4E constructs. pHA-eIF4E was a gift from Dong-Er Zhang (UCSD, La Jolla, California, USA) (Addgene plasmid catalog 17343). The S209D and wobble mutations were generated via site-directed mutagenesis (QuikChange II XL Site-Directed Mutagenesis Kit, Stratagene). WT and mutant cDNAs were subsequently cloned into the pHAGE-Flag-HA mammalian lentiviral expression vector.

Binding constant measurements and calculations. Binding of epitope-tagged MNK1 and MNK2 to immobilized ligand was measured as a function of unlinked test compound concentration using methods described previously (26). Cabozantinib dose-response curves were graphed using nonlinear least-square fit with the LevenbergMarquardt algorithm. Determining kd based on the dose response curves used the Hill equation with the Hill slope set to -1 .

qPCR. RNA was isolated using QIAGEN RNeasy kit. $50 \mathrm{ng}$ of total RNA was used for each reaction, and cDNA synthesis and PCR amplification were performed using the qScript One-Step SYBR Green Kit for iQ (Quanta Biosciences). Quantitative PCR (qPCR) reactions were run using a Bio-Rad iCycler iQ thermal cycler. Samples were run in triplicate, and expression levels were determined based on a standard curve run with each primer set; levels were normalized to an internal control, GAPDH. The following are the primer sequences used: GAPDH; for: 5'-CATGTTCGTCATGGGTGTGAACCA-3', rev: 5'-ATGGCATGGACTGTGGTCATGAGT-3' and MKNK2; for: 5'-AGCCGAACTTCAGGGTTTC-3', rev: 5'-AAGTCAGAGTCTCCGTGGT-3'.

In vivo drug treatments and dosing schedule. C57BL/6-Trp53 $3^{\text {tmITyj }}$ $N f 1^{t m 1 T y j}$ (NPcis) mice were bred and maintained as previously described (24). Mice were palpated to detect tumors starting at 2 months of age, and treatment commenced when tumors reached 200 to $1,000 \mathrm{~mm}^{3}$. Mice were treated daily with cabozantinib by oral gavage at $12.3 \mathrm{mg} / \mathrm{kg}$. Cabozantinib was prepared in 65\% D5W/30\% propylene glycol/5\% Tween- 80 and was briefly vortexed followed by sonication for 2 minutes. PD901 was administered at $1.5 \mathrm{mg} / \mathrm{kg}$ once per day immediately prior to cabozantinib administration by oral gavage, and PD901 was prepared as previously described (27). To track changes in tumor volume, tumor size was measured at day 0 and subsequently every 2 to 3 days by vernier calipers; volume was calculated using the standard formula $\mathrm{L} \times \mathrm{W}^{2} \times 0.52$.

Statistics. For quantitative measurements, graphs represent mean \pm SD. Where indicated, data are presented as fold change or $\log _{2}$ fold change over initial measurements. Changes in tumor volume are presented in a waterfall plot with each bar representing the change in tumor volume of an individual animal in the study. ANOVA followed by Bonferroni's multiple comparison tests, 2-tailed unpaired $t$ tests, and Mann-Whitney $U$ tests was used to compare data sets where indicated, and $P$ values are shown. A $P$ value less than or equal to 0.05 was considered significant. Data were graphed and analyzed using GraphPad Prism v.6.

Study approval. Animal procedures were approved by the Center for Animal and Comparative Medicine at Harvard Medical School in accordance with the NIH Guide for the Care and Use of Laboratory Animals (National Academies Press. 2011.) and the Animal Welfare Act. Consent was obtained for human tissue acquisition according to IRBapproved protocol (S52563) and local ethical committees.

\section{Author contributions}

RL, RI, OM, ALM, NW, BMK, SCBY, and JRG performed the experiments. RL, BMK, JRG, and KC designed and interpreted the experiments. EL contributed advice and provided human tumor samples. RL, BMK, JRG, and KC wrote and edited the manuscript.

\section{Acknowledgments}

K. Cichowski, R. Lock, and this work were supported by a grant from the Department of Defense (W81XWH-13-1-0044), the Children's Tumor Foundation (CTF), and the National Cancer Institute (R01CA111754). R. Lock was supported by an American Cancer Society-New England Division-Ellison Foundation postdoctoral fellowship (PF-15-127-01-CDD) and by a CTF Young Investigator Award (2012-01-023).

Address correspondence to: Karen Cichowski, Brigham \& Women's Hospital, 77 Avenue Louis Pasteur, NRB 0458C, Boston, Massachusetts 02115, USA. Phone: 617.525.4722; E-mail: kcichowski@rics.bwh.harvard.edu. 
1. Maertens $\mathrm{O}$, Cichowski K. An expanding role for RAS GTPase activating proteins (RAS GAPs) in cancer. Adv Biol Regul. 2014;55:1-14.

2. Martin GA, et al. The GAP-related domain of the neurofibromatosis type 1 gene product interacts with ras p21. Cell. 1990;63(4):843-849.

3. Ballester R, et al. The NF1 locus encodes a protein functionally related to mammalian GAP and yeast IRA proteins. Cell.1990;63(4):851-859.

4. Bollag G, et al. Loss of NF1 results in activation of the Ras signaling pathway and leads to aberrant growth in haematopoietic cells. Nat Genet. 1996;12(2):144-148.

5. Johannessen CM, et al. TORC1 is essential for NF1-associated malignancies. Curr Biol. 2008;18(1):56-62.

6. Chang T, et al. Sustained MEK inhibition abrogates myeloproliferative disease in Nf1 mutant mice. J Clin Invest. 2013;123(1):335-339.

7. Jessen WJ, et al. MEK inhibition exhibits efficacy in human and mouse neurofibromatosis tumors. JClin Invest. 2013;123(1):340-347.

8. Riccardi VM. Neurofibromatosis: Phenotype, Natural History, And Pathogenesis. Baltimore, Maryland, USA: The Johns Hopkins University Press; 1992.

9. Zehou O, et al. Chemotherapy for the treatment of malignant peripheral nerve sheath tumors in neurofibromatosis 1: a 10-year institutional review. Orphanet J Rare Dis. 2013;8:127.

10. Johannessen CM, Reczek EE, James MF, Brems H, Legius E, Cichowski K. The NF1 tumor suppressor critically regulates TSC2 and MTOR. Proc Natl Acad Sci U S A. 2005;102(24):8573-8578.

11. Malone CF, Fromm JA, Maertens O, DeRaedt T,
Ingraham R, Cichowski K. Defining key signaling nodes and therapeutic biomarkers in NF1-mutant cancers. Cancer Discov. 2014;4(9):1062-1073.

12. Pelletier J, Graff J, Ruggero D, Sonenberg N. Targeting the eIF $4 \mathrm{~F}$ translation initiation complex: a critical nexus for cancer development. Cancer Res. 2015;75(2):250-263.

13. Buxade M, Parra-Palau JL, Proud CG. The Mnks: MAP kinase-interacting kinases (MAP kinase signal-integrating kinases). Front Biosci. 2008;13:5359-5373.

14. Truitt ML, et al. Differential requirements for eIF4E dose in normal development and cancer. Cell. 2015;162(1):59-71.

15. Ueda T, Watanabe-Fukunaga R, Fukuyama H, Nagata S, Fukunaga R. Mnk2 and Mnk1 are essential for constitutive and inducible phosphorylation of eukaryotic initiation factor $4 \mathrm{E}$ but not for cell growth or development. Mol Cell Biol. 2004;24(15):6539-6549.

16. Furic L, et al. eIF4E phosphorylation promotes tumorigenesis and is associated with prostate cancer progression. Proc Natl Acad Sci U S A. 2010;107(32):14134-14139.

17. Ueda T, et al. Combined deficiency for MAP kinaseinteracting kinase 1 and 2 (Mnk1 and Mnk2) delays tumor development. Proc Natl Acad SciU S A. 2010;107(32):13984-13990.

18. Wendel HG, et al. Dissecting eIF4E action in tumorigenesis. Genes Dev. 2007; 21(24):3232-3237.

19. Knauf U, Tschopp C, Gram H. Negative regulation of protein translation by mitogen-activated protein kinase-interacting kinases 1 and 2. Mol Cell Biol. 2001;21(16):5500-5511.
20. Konicek BW, et al. Therapeutic inhibition of MAP kinase interacting kinase blocks eukaryotic initiation factor $4 \mathrm{E}$ phosphorylation and suppresses outgrowth of experimental lung metastases. Cancer Res. 2011;71(5):1849-1857.

21. Yan SB, et al. LY2801653 is an orally bioavailable multi-kinase inhibitor with potent activity against MET, MST1R, and other oncoproteins, and displays anti-tumor activities in mouse xenograft models. Invest New Drugs. 2013;31(4):833-844.

22. Yakes FM, et al. Cabozantinib (XL184), a novel MET and VEGFR2 inhibitor, simultaneously suppresses metastasis, angiogenesis, and tumor growth. Mol Cancer Ther. 2011;10(12):2298-2308.

23. Zou HY, et al. Sensitivity of selected human tumor models to PF-04217903, a novel selective c-Met kinase inhibitor. Mol Cancer Ther. 2012;11(4):1036-1047.

24. Cichowski K, et al. Mouse models of tumor development in neurofibromatosis type 1. Science. 1999;286(5447):2172-2176.

25. Awad MM, Shaw AT. ALK inhibitors in non-small cell lung cancer: crizotinib and beyond. Clin $A d v$ Hematol Oncol. 2014;12(7):429-439.

26. Fabian MA, et al. A small molecule-kinase interaction map for clinical kinase inhibitors. Nat Biotechnol. 2005;23(3):329-336.

27. Brown AP, Carlson TC, Loi CM, Graziano MJ. Pharmacodynamic and toxicokinetic evaluation of the novel MEK inhibitor, PD0325901, in the rat following oral and intravenous administration. Cancer Chemother Pharmacol. 2007;59(5):671-679. 